\title{
E04 | Seismic-to-simulation Integration for Autonomous Fields and Real- time Monitoring: The Advantage of Gigacell Simulation
}

\author{
By Jorge Pita \& Ali Dogru (Saudi Aramco)
}

\begin{abstract}
:
Reservoir simulation technology capable of handling billions of cells makes it possible to model reservoirs with unprecedented geological and fluid characterization detail, either minimizing or eliminating upscaling and its adverse effects. The speed of these simulations, aided by new computer technologies, will enable us to make inroads into new paradigms, such as autonomous fields coupled with seismic-to-simulation integration.
\end{abstract}

Faster computations will lead us into real-time simulations, where simulations will run concurrently with Intelligent-Field data acquisition. These simulations will be auto-calibrated with real-time data and will advise on mitigating actions before undesirable reservoir conditions occur. Our current implementations enable the reservoir simulator to provide voice warnings while dynamically using a petro-elastic model to predict seismic and electromagnetic properties. The next step is to compare this simulation with live continuous monitoring data (Figure 1) and potentially improve the model over time.

Today, these predictions are being used to rank reservoir monitoring technologies and design the best location and time window intervals for continuous monitoring. Once in place, these continuous measurements will help improve reservoir models by calibrating the predicted with measured values, effectively "closing the loop" of seismic and simulation integration.

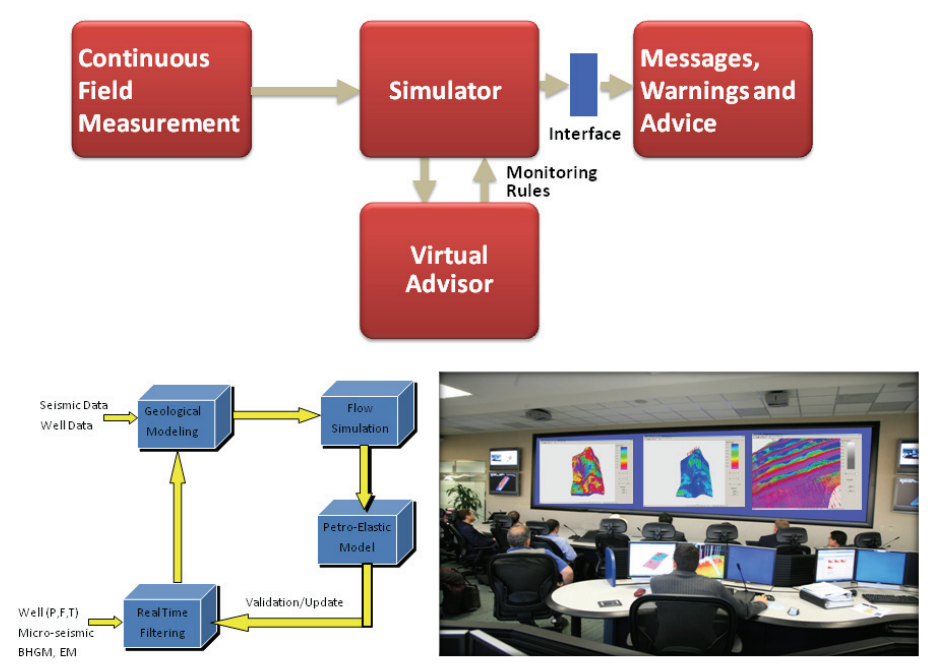

Figure 1: Seismic to Simulation Integration in Autonomous Fields 